\title{
Abordajes de la Lectura por parte de Sordos en Contextos Bilingües en Países de Habla Hispana
}

\author{
Deaf People Reading in Bilingual Contexts in Spanish Speaking \\ Countries
}

\author{
María Virginia Hael * \\ Consejo Nacional de Investigaciones Científicas y Técnicas, Instituto del Desarrollo Humano, \\ Universidad Nacional de General Sarmiento, Argentina
}

\begin{abstract}
Este trabajo indaga acerca de los aportes teóricos vinculados con los procesos de lectura en sordos, sus problemáticas, sus avances, y las consiguientes prácticas educativas. Para ello, se analizaron artículos teóricos y de investigación empírica en lengua española. A partir del estudio hemos podido comprobar que el bilingüismo es la corriente más difundida en la actualidad y que, a su vez, dentro de esta teoría existen diferentes concepciones sobre la lectura: una que aboga por el aprendizaje del español como segunda lengua solo en su variante escrita, sin mediación de la oralidad y otra que, por el contrario, considera que el español oral facilita la adquisición de la lectura en segunda lengua.De nuestra observación se desprende que la adquisición de vocabulario, la conciencia fonológica (en su variante visual) y la sintaxis son elementos fundamentales para el desarrollo de la lectura en sordos, que deberían ser ejercitados de manera más sistemático en el abordaje que actualmente se realiza desde el enfoque bilingüe. Además, proponemos la logogenia como un método alternativo y accesorio para el entrenamiento de habilidades sintácticas en el procesamiento de la lectura en el caso de las personas sordas. Creemos que el aprendizaje de la lectura y la escritura por parte de los miembros de esta comunidad es importante para la plena apropiación de conocimientos escolares.
\end{abstract}

Descriptores: Sordo; Lectura; Bilingüismo; Sintaxis; Educación.

This paper investigates different theories about the reading processes in deaf people, their problems, their advances and educational practices. For that, we have analyzed theoretical and empirical research papers in Spanish. After our analysis, we arrived at the conclusion that nowadays bilingualism is the main school of thought, even when there are different ideas about reading inside it: one of those ideas is about the acquisition of Spanish as a foreign language not only in the writing skills, without any participation of the oral language and other that, in opposition, consider that oral Spanish make it easier to learn how to read and write in that language. After this study, we think that vocabulary acquisition, phonological consciousness (in its visual variant), and syntaxes are important to develop reading skills in deaf people, that should be trained more systematically by the bilingual perspective. We also propose that logogenia is possibility of an alternative and accessory method for the syntax skills training involved in every reading and writing process. We also think that it is very important for deaf people to learn how to read and write in Spanish language, so they would have a better and fuller acquisition of scholar knowledge.

Keywords: Deaf; Reading; Bilingualism; Syntax; Education.

*Contacto: virginiahal@gmail.com

ISSN: 0718-7378

www.rinace.net/rlei/
Recibido: 15/05/2018

$1^{a}$ Evaluación: 10/07/2018

Aceptado: 20/08/2018 


\section{Introducción}

La comprensión y producción de textos por parte de sordos es un tema de amplio debate y preocupación entre los docentes que deben asumir la responsabilidad de enseñar lectura y escritura a esta población. Al ser estas habilidades la base a través de la que se sustenta buena parte de la construcción del conocimiento escolar es fundamental su aprendizaje para poder acceder a los contenidos de todas las asignaturas de la educación primaria, secundaria y superior.

Es a partir de la reflexión acerca de las dificultades que presentan los sordos en este aspecto que surgen interrogantes vinculados con los abordajes que la educación ofrece al respecto. En este sentido, el objetivo de este trabajo es indagar acerca de los aportes teóricos, y las consiguientes puestas en práctica a través de la educación, en relación con los procesos de lectura en la comunidad sorda, sus problemáticas y sus avances. Para ello, hemos analizado artículos de investigación producidos en lengua española acerca de esta temática.

\section{1. ¿Qué sabemos acerca de las dificultades lectoras en esta población?}

Una de las mayores dificultades a la hora de abordar la lectura por parte de niños sordos es la escasa cantidad de vocabulario en español que conocen en relación con sus pares oyentes (Conrad, 1979; Silverman-Dresner y Guilfyle, 1972; Trybus y Karchmer, 1977; citado en Augusto et al., 2002). Se constata, además, que entre el vocabulario utilizado por los niños sordos priman los sustantivos y verbos -a menudo sin conjugar- y es menor la inclusión de pronombres, preposiciones y adjetivos (Augusto et al, 2002; Domínguez Gutiérrez, 2003). Además, los sordos tienden a fijarse más en palabras familiares de un texto que en las palabras realmente clave, lo que constituiría a un obstáculo más en la comprensión lectora (Domínguez Gutiérrez, 2003; Domínguez Gutiérrez y Soriano Bozalongo, 2009). En estrecha relación con lo anterior, tienen dificultades en las habilidades sintácticas, mayormente en las estructuras con objeto directo, conjunciones, oraciones pasivas y cláusulas de relativo (Domínguez Gutiérrez, 2003).

Las investigaciones indican que la mayor parte de los sordos no alcanza niveles funcionales de lectura, con retrasos de siete u ocho años y llegando a un nivel general de comprensión lectora de niños de segundo y tercer año de escuela primaria. En esta línea de estudios, una investigación experimental con grupo de control de niños oyentes de diferentes cursos de primaria llegó a la conclusión de que los sordos que son considerados buenos lectores alcanzan un nivel de comprensión morfosintáctica equivalente a la de niños de quinto año y emplean estrategias ortográficas en la comprensión lectora para suplir la falta de información fonológica, lo que implica una mayor cantidad de tiempo de procesamiento lingüístico. El empleo de estrategias semánticas parece ser característico de la lectura de sordos en general, no solamente entre los malos lectores (Domínguez Gutiérrez y Soriano Bozalongo, 2009).

Para Svartholm y González (2012), desde un enfoque bilingüe -que analizaremos a continuación-, los errores que cometen usualmente los sordos al leer y escribir en español son propios de cualquier persona que aprende una segunda lengua y se vinculan con estrategias tales como simplificación, sobregeneralización y el aprendizaje holístico de 
palabras y frases y que explicaría el porqué de las dificultades de esta población con las preposiciones, las inflexiones y el uso no literal del lenguaje. Dentro de esta línea, consideramos que sería interesante analizar cuáles son las estrategias docentes para mejorar esos aspectos del aprendizaje de cualquier segunda lengua y evaluar la posibilidad de implementarlas con la comunidad sorda.

\section{El enfoque bilingüe bicultural}

Actualmente prima entre las políticas lingüísticas de diversos países de habla hispana el enfoque bilingüe bicultural de la educación del sordo por sobre las orientaciones oralistas que tuvieron supremacía en décadas pasadas. Es por este motivo que nos abocaremos a la revisión bibliográfica sobre este a fin de conocer cuáles son las investigaciones actuales acerca de esta propuesta.

En primera instancia, es importante definir qué es el bilingüismo y qué implicancias tiene en el caso de su abordaje desde la sordera. Para Skliar (1998), el bilingüismo aplicado a la educación del sordo tiene que aludir específicamente al derecho que tienen los sujetos con una lengua minoritaria a ser educados en esa lengua. En este sentido, nos parece importante aclarar que la lengua de señas es una lengua completa, natural y compleja. Se opone, entonces, a las corrientes oralista y neo-oralista ${ }^{1}$ que dejan de lado la lengua de señas para enfocarse únicamente en la adquisición primordialmente oral -y a partir de esta, escrita- de la lengua española². En relación con esto, Domínguez Gutiérrez (2003) sostiene que la educación bilingüe favorece en mayor medida el aprendizaje de la lectura en niños sordos, en contraposición con las opciones de educación monolingüe -basadas en la adquisición del español como única lengua tanto en su nivel oral como escrito- que entrenan simultáneamente el aprendizaje de la lengua oral y la escritura. En general, aquellos que acceden a una educación monolingüe poseen menos conocimiento sobre el mundo y menor vocabulario que aquellos que acceden a una educación bilingüe. En contextos que solo propician la oralización de sordos, Domínguez Gutiérrez(2003) se refiere específicamente al poco contacto con lo escrito que tienen estos niños. En cambio, en hogares en los que se habla lengua de señas, los niños parecen tener más relación con lo escrito a través de la lectura de cuentos infantiles, el acceso a libros, adaptando la posición de las señas de manera cercana al texto para lograr la atención de los niños pequeños.

Para Cummins (1984) se puede encontrar tres tipos de bilingüismo. En primer lugar, un bilingüismo limitado, en el que la competencia en ambas lenguas es pobre. En segundo lugar, un bilingüismo desequilibrado, en el que existe una buena competencia solamente en una de las dos lenguas. En tercer lugar, un bilingüismo equilibrado en el que la competencia se desarrolla de manera adecuada para ambas lenguas. Herrera Fernández (2009) menciona que desde los abordajes del bilingüismo se sostiene que las habilidades lectoras y escritoras en L1 inciden positivamente en dichas habilidades en L2. A partir de esta noción general, la autora sostiene que el bilingüismo en el caso de los sordos adquiere

\footnotetext{
${ }^{1}$ Peluso y Vallarino (2014) hacen referencia a este término implicando el oralismo derivado de los implantes cocleares, que muchas veces considera que, por el hecho de haber introducido el implante coclear en el sordo, se convierte automáticamente en oyente y se lo educa solamente en lengua oral -y escrita- dejando de lado la lengua de señas.

${ }^{2}$ En el caso de los sordos de países hispanohablantes.

${ }^{3}$ Nos referimos aquí al bilingüismo en términos generales, no en relación con la educación del sordo.
} 
características específicas que impiden su comparación con otros tipos. Con respecto a esto, menciona que entre ambas lenguas existen diferencias notables: en primer lugar, los modos de transmisión, percepción y expresión, que en el caso de la lengua de señas es mediante el sistema visual e implica en gran medida el aspecto kinésico y en la lengua hablada -en nuestro caso, el español- se realiza a través del sistema auditivo e implica movimientos bucales y labiales. Recalca también la dificultad que implica para el alumno sordo adentrarse en la cultura escrita de una segunda lengua cuando en la mayoría de los casos no tienen consolidada su primera lengua.

En cuanto al bilingüismo en contextos de sordera, Domínguez Gutiérrez (2003) menciona dos abordajes: en primera instancia, una concepción que indica que el aprendizaje del español como lengua segunda debe hacerse únicamente en su versión escrita, es decir, consideran al español escrito como segunda lengua. Una concepción más amplia, pretende la enseñanza de las versiones tanto escrita como oral como segunda lengua ya que considera que la adquisición de la oralidad va a desarrollar una mejor posibilidad de lectura labio facial, que con ayuda de la palabra complementada colabora en la formación de conciencia fonológica en esta población, que a su vez facilita la comprensión de la palabra escrita.

Para Sánchez Avedaño (2005) en la educación bilingüe del sordo es primordial que se desarrolle de manera completa la lengua de señas, que además debe ser estudiada por el sordo en todos sus niveles: fonológico, morfológico, sintáctico y discursivo -para lo que se debe llevar a cabo un proceso de estandarización- y debe ser el vehículo de enseñanza del español. Agrega que, si la situación real del sordo fuese el desarrollo natural de la lengua de señas a través de su exposición al medio lingüístico desde el nacimiento, el bilingüismo sería de más fácil abordaje. Sánchez Avedaño (2005) aboga por un bilingüismo basado principalmente en la decodificación del código escrito -y no el oral- del español. A pesar de esto, destaca que una de las mayores dificultades en este sentido es que los niños deben ser alfabetizados en una segunda lengua, es decir que deben aprender a leer y a escribir no en su lengua materna sino en su segunda lengua. Así también, destaca que es sumamente necesario que los docentes de sordos cuenten con formación en lengua de señas, didáctica de las segundas lenguas y en español. En este aspecto coinciden Lissi, Svartholm y González (2012) y agregan la importancia de la inclusión de adultos sordos en la docencia.

Por su parte, y coincidiendo con los planteos anteriores, Skliar (1998) sostiene que en la educación bilingüe y bicultural del sordo es fundamental la apropiación y la valorización de la propia lengua por sí misma y también en contraste con la segunda lengua, además de que se debe sostener la lengua de señas a lo largo de todo el trayecto educativo y debe difundírsela más allá de los límites de la escuela, así como la cultura sorda. En términos de participación, propone que los sordos deben poder tener acceso a la información, al mundo laboral y deben poder participar plenamente como ciudadanos.

En relación con la línea más abierta, Grosjean (2000) piensa que, para el sordo, crecer bilingüe es un derecho que implica el conocimiento profundo de la lengua de señas y la lengua oral -en su versión escrita y en lo posible también hablada- a fin de que alcance un desarrollo completo de sus habilidades lingüísticas, cognitivas y sociales.

A continuación, desarrollaremos con mayor profundidad los avances y las propuestas desde el bilingüismo que propicia tanto la adquisición de la oralidad como de la escritura. Los aportes de la conciencia fonológica en el abordaje de la lectura y la escritura de sordos 
en contextos bilingües. En relación con el segundo tipo de bilingüismo propuesto por Domínguez Gutiérrez (2003), que acepta la influencia de la lengua oral para desarrollar la escritura, podemos mencionar las siguientes propuestas teóricas.

Herrera Fernández (2009) retoma la noción de bilingüismo equilibrado propuesto por Cumminis (1984) y sostiene firmemente que, para lograrlo en el caso de alumnos sordos, es fundamental estimular tanto la lengua de señas como la lengua oral. La autora destaca la importancia de la información fonológica para el desarrollo de la decodificación en sordos, e incluso subraya que los sordos que son considerados buenos lectores son aquellos que hacen uso de códigos fonológicos. Dentro de las posibilidades en el modelo de educación bilingüe tradicional -que prioriza la adquisición del español escrito y no el hablado como segunda lengua- destaca la dactilología como sostén de la decodificación. Este sistema permite que los niños puedan usar la lengua de signos para escribir en una segunda lengua (Domínguez Gutiérrez y Alegría, 2009). Se trata de una representación del alfabeto de manera manual en la que existe una correlación entre una seña del alfabeto manual y una forma ortográfica del español.

En un estudio acerca de las dificultades lectoras en esta población, Augusto y otros (2002) llegan a la conclusión de que las habilidades metafonológicas son importantes para el desarrollo de la comprensión lectora, por lo que defienden su desarrollo en niños sordos sin restar importancia al lenguaje de señas como transmisor de cultura y conocimiento defendiendo, inclusive, la necesidad de su desarrollo en etapas previas a la escolarización por su importancia a la hora de comunicar-. Domínguez Gutiérrez y Alegría (2009) coinciden en el hecho de que las habilidades fonológicas tienen un correlato en la comprensión lectora. A los factores que determinan el buen desempeño lector le añaden la competencia lingüística: en este sentido, explican que conocer todas -o la mayor parte de- las palabras que componen una frase y la sintaxis que las organiza facilita la comprensión lectora. Si bien no se trata de un aspecto específico de la lectura -porque la competencia lingüística también incide sobre la comprensión oral-, sí es indispensable para la comprensión de lo escrito.

A partir de la noción de que la conversión grafema-fonema es lo que permite identificar todas las palabras del español escrito -lo que destaca la importancia fundamental de la fonología en el procesamiento de la lectura, y la dificultad que implica intentar leer sin esta posibilidad-, los autores proponen que existe la posibilidad de generar conciencia fonológica sin audición y la vinculan, fundamentalmente, con la lectura labio facial que no solamente es empleada en el caso de personas sordas sino también oyentes (Alegría, 2003). Es necesario resaltar que la lectura labio facial por sí misma es insuficiente para activar el léxico interno en los sordos, ya que hay fonemas que pueden resultar ambiguos y, por lo tanto, activar palabras equivocadas. Ante esta situación se han desarrollado sistemas para desambiguar los fonemas que pueden generar complicaciones, uno de los más importantes es la palabra complementada. Se trata de un agregado a la lectura labio facial que provee claves manuales -que se ejecutan en diferentes posiciones alrededor de la boca- para desambiguar fonemas que pueden resultar ambiguos. Domínguez Gutiérrez y Alegría (2009) destacan que es posible desarrollar conciencia fonológica a través de su enseñanza específica a partir de la combinación de ambos sistemas, que facilita el aprendizaje de la lectura.

Es necesario mencionar, asimismo, que Martínez de Antoñana y Augusto Landa (2002) afirman que las dificultades lectoras en niños sordos se basan, primordialmente, en su 
desconocimiento de la lengua oral. Los autores presentan resultados de un estudio llevado a cabo con el objeto de conocer qué ruta ${ }^{4}$ utilizan sordos profundos en la lectura en voz alta de palabras y pseudopalabras, utilizando un grupo control de sujetos oyentes. Llegan a la conclusión de que los oyentes emplean ambas rutas de manera complementaria, mientras que los sordos se apoyan fundamentalmente en la vía fonológica, aunque esto puede variar según la etapa de escolarización en la que se encuentren.

Figueroa y Lissi (2005) reflexionan acerca de cuáles son las alternativas que utilizan los sordos al no tener la conciencia fonológica en su variante clásica. Destacan, al igual que otros autores relevados en este artículo, al deletreo manual como una forma de recodificación del lenguaje escrito que recuperaría los fonemas. A su vez, hacen mención de otras estrategias basadas en lo visual, tales como la lectura labial y la palabra complementada. A pesar de ello, consideran que las investigaciones llevadas a cabo en este sentido no aportan datos que prueben de manera precisa la incidencia de estas estrategias en la comprensión lectora. Por otra parte, se refieren a estudios que establecen una correlación entre una elevada competencia en lenguaje de señas y un buen nivel lector. Al igual que respecto de las investigaciones sobre conciencia fonológica, Figueroa y Lissi (2005) postulan que los estudios acerca de la incidencia de la lengua de señas en el desempeño lector no proveen información acerca de cómo se ejecutaría dicha relación entre ambas lenguas. Ante esta situación, promueven, por ejemplo, que se realicen mayores investigaciones vinculadas con la estrategia de encadenamiento, que se basa en una especie de gramática comparativa entre la lengua de señas y el lenguaje escrito. Con respecto a este asunto, como el español y la lengua de señas son dos lenguas muy diferentes en sus niveles morfológico, sintáctico, y fundamentalmente, fonológico, Domínguez Gutiérrez y Alegría (2009), consideran que la relación entre L1 y L2 se da de manera distinta a lo que ocurre habitualmente: creen que el mayor aporte que otorga el manejo de la lengua de señas para el aprendizaje del español es el bagaje de conocimiento de mundo y de temas concretos. En este sentido, los docentes pueden explicar más fácilmente conceptos, definiciones, palabras desconocidas del español, haciendo uso de la lengua de señas. A esto se suma que ante niños competentes en lengua de señas es más sencillo establecer comparaciones entre las estructuras gramaticales de esta lengua y del español.

\section{El implante coclear dentro del marco de la educación bilingüe bicultural}

Un aspecto controvertido dentro de la educación y la salud del sordo es el implante coclear. Generalmente se lo asocia con tendencias oralistas, que consideran que con el implante colcear el sordo se transforma en oyente y que dejan de lado la lengua de señas en detrimento de la lengua oral. A pesar de esto, algunas corrientes de educación bilingüe consideran que el implante coclear puede tener incidencia positiva en los procesos lectores de la población sorda.

Entre ellos, Peluso y Vallarino (2014) estudian cómo funciona el bilingüismo en escuelas primarias para sordos en Uruguay, contraponiéndolas a las clases para sordos que se imparten en escuelas comunes y a la situación de los sordos con implantes cocleares

\footnotetext{
${ }^{4}$ Léxica o fonológica (Ellis y Young, 1992).
} 
integrados en escuela común. Rescatan que en las escuelas para sordos la comunidad tiene una participación muy activa que implica no solo la transmisión de la lengua de señas sino también de la cultura sorda e incide en el sentimiento de identidad de los niños. En estas escuelas, los niños aprenden lengua de señas como primera lengua y el español en su variante escrita como segunda lengua. Así también, ofrecen cursos y talleres de lengua de señas a padres y familiares recalcando en la necesidad de que la aprendan para poder comunicarse y generar lazos auténticos con sus hijos. En cuanto a la situación de los sordos implantados y oralizados en contextos monolingües, realizan un relevamiento acerca del desempeño lector de esos estudiantes y llegan a la conclusión de que la mitad de los sordos oralizados, a pesar del implante coclear, no produce ni comprende lenguaje oral y la otra mitad lo logra de manera muy pobre. A pesar de esto, entienden que, en un contexto bilingüe, de respeto por la lengua y la cultura sorda, el implante coclear puede generar ciertas ventajas a la hora de adquirir el español como segunda lengua -que puede tener incidencia tanto en la comunicación oral como en la comprensión y producción de textos escritos-.

Domínguez Gutiérrez y Alegría (2009) mencionan estudios relacionados con el implante coclear realizado precozmente (aproximadamente a los 27 meses) unido al uso de la palabra complementada. Los casos analizados indican resultados en lectura similares entre los sordos con estas posibilidades y los oyentes de la misma franja etaria. Esto indicaría que el implante coclear sumado al método de la palabra complementada en contextos bilingües sería positivo para el aprendizaje de la lectura.

Domínguez Gutiérrez, Pérez Sánchez y Soriano Borzalongo (2007) realizaron un estudio contrastivo acerca del desempeño lector por parte de sordos con implante coclear y sordos sin implante coclear en el marco de una educación bilingüe, y utilizaron un grupo de control conformado por oyentes. En el caso del primer grupo también diferenciaron entre aquellos a los que se los implantó de manera temprana y aquellos a los que se los implantó de manera tardía. Las pruebas contemplaron variables tales como la eficiencia lectora, las estrategias sintácticas, decisión ortográfica y pruebas metafonológicas. Los oyentes tuvieron mejor desempeño que los sordos en todas las pruebas, y entre los sordos, resolvieron de mejor manera aquellos con implante coclear temprano que aquellos con implante coclear tardío y todo el grupo de sordos implantados tuvo mejor desempeño que los sordos sin implante coclear. La prueba en la que peor desempeño demostraron todos los sordos fue en la de estrategias sintácticas. A partir de los resultados de su estudio, los autores sugieren que las instituciones educativas deben enfocarse en desarrollar estrategias de enseñanza de los aspectos sintácticos a esta población.

Estas investigaciones parecerían indicar que el implante coclear puede tener una influencia positiva en los procesos de lectura y escritura de la población sorda, siempre y cuando se realice en contextos bilingües en los que la lengua de señas sea respetada como primera lengua y el español tanto oral como escrito sea considerado segunda lengua.

\section{La situación de la educación bilingüe del sordo en países de Latinoamérica}

En este apartado hacemos un recorrido por algunas experiencias de educación bilingüe y bicultural de los sordos llevadas a cabo en países de Latinoamérica a fin de conocer la 
situación actual de la educación de esta población y cómo se llevan a cabo las aplicaciones de esta corriente.

En primer lugar, podemos hacer mención del estudio llevado a cabo por Lissi, Svartholm y González (2012) en el que destacan las ventajas del modelo sueco de educación bilingüe que se orienta a que los sordos, tras diez años de escolaridad obligatoria, sean competentes tanto en lengua de señas como en sueco (e incluso tienen inglés en su currícula). A partir de la exposición de las ventajas de este modelo, entre las que se destaca que los sordos terminan la escolaridad obligatoria con un desempeño en lengua sueca de un $59 \%$, las autoras reflexionan acerca de lo que sucede en Chile. Mencionan que, si bien ha habido una progresiva incorporación de la lengua de señas en las escuelas de sordos, no existe un verdadero modelo bilingüe sistemático. Las autoras presentan resultados de la observación de las actividades y estrategias educativas generadas en una escuela bilingüe/bicultural chilena y el desempeño en lengua de señas y lengua escrita obtenido por los alumnos. Muestran ejemplos de cómo se abordan diferentes situaciones de lectura, entre ellas la lectura en lengua de señas -los estudiantes leen un fragmento y lo señan y así sucesivamente hasta completar el texto-, en el que deben respetar la estructura de la LS. La lectura en este caso no debe ser palabra por palabra, sino que deben considerarse unidades de sentido completas para poder expresarlas en LS. Otra de las estrategias que pudieron observar fue la de asegurar la comprensión del texto, para lo cual los docentes explican, previamente a la lectura, el tema principal del texto, lo ponen en contexto y explican el significado de palabras que son desconocidas para los niños. Este tipo de actividades sirve también para poder especificar en contexto algunas diferencias gramaticales específicas entre la lengua de señas y el español escrito. Las autoras consideran que en los contextos bilingües es fundamental que los niños estudien el español como segunda lengua y la lengua de señas como una asignatura, además de que sea la lengua principal de comunicación.

Larrinaga y Peluso (2007) investigaron cómo se lleva a cabo la educación bilingüe en una escuela secundaria en Uruguay. Observan, en primera instancia, que se imparte lengua de señas como una asignatura obligatoria y que, a su vez, también se dan clases de español como segunda lengua. Encuentran también, tres posiciones discursivas dentro del aula: por un lado, docentes de todas las materias -menos de LSU- que dictan sus clases en español, desconociendo la lengua de señas y situándose en el lugar de "saber" los contenidos curriculares. En segundo lugar, los intérpretes que son quienes vehiculizan el saber curricular, a pesar de no tener que conocer los contenidos curriculares. Y, por último, los alumnos sordos, que necesitan conocer los contenidos de las asignaturas y, a su vez, deben aprender español en su variante escrita. En este contexto, los docentes manifiestan dificultades por los impedimentos que el bajo desempeño lector de los alumnos genera: en muchas ocasiones, más que impartir conocimientos disciplinares, deben ejercer como docentes de lengua. Ante esto, los autores desarrollan una propuesta en la que desde cada asignatura se trabaje con textos disciplinares desde la metodología de enseñanza del español como segunda lengua, a fin de que los docentes puedan impartir los conocimientos necesarios de cada materia y, a su vez, estimular la comprensión escrita en español por parte de los alumnos.

Henao Álvarez, Ramírez Salazar y Medina Medina (2003) realizan una experiencia multimedial en un entorno escolar bilingüe en Medellín, Colombia. Para ello desarrollaron un programa tendiente a mejorar habilidades de lectura en contexto y en lengua de señas colombiana. El estudio es experimental con pre prueba, post prueba y grupo de control. 
Tras un tiempo de cuatro meses de uso de la plataforma multimedial y contrastando las prepruebas y las postpruebas, llegan a la conclusión de que la intervención tuvo influencia positiva en estrategias de búsqueda de información en textos de diferente tipología, en producción de respuestas literales sobre un texto breve, la habilidad de comprender y ejecutar órdenes escritas, la selección correcta de palabras que completan frases, la vinculación adecuada entre señas y palabras escritas y la capacidad de explicar en lengua de señas un conjunto de frases escritas, entre otras. Los alumnos del grupo experimental tuvieron un desempeño mucho mejor que los del grupo control. Recalcan, además, en la importancia de la participación de adultos sordos en la educación de niños en contextos bilingües.

Zambrano (2002) informa acerca de una experiencia con el segundo año de Ciencias, perteneciente a la Unidad Educativa Nacional de Educación Especial "Maracay", Venezuela. La investigación tenía como finalidad conocer la competencia lectora de los estudiantes que egresan de tal institución. Para ello, desarrolló una serie de pruebas que implicaba la lectura de un texto expositivo de Biología y de una noticia del periódico local y la resolución de preguntas que, o bien requerían respuestas literales o bien requerían de operaciones intelectuales aplicadas a la información, tales como comparar o analizar. Llega a la conclusión de que, en ambos textos, el primer tipo de preguntas no generaba mayores dificultades, mientras que el segundo tipo de preguntas sí lo hacía. Destaca también que ninguna respuesta se presentó con sintaxis competente del español, sino que más bien parecía ser una traducción literal, palabra por palabra, de la lengua de señas al español.

Por su parte, Cruz Aldrete (2009) investiga acerca de la situación de la educación de niños sordos indígenas en México citando el caso de la comunidad de Chicán de Yucatán, donde los sordos dominan el maya oral y la Lengua de Señas Maya, utilizada tanto por sordos como por oyentes para la comunicación en la comunidad. Considera que en los casos de los sordos pertenecientes a comunidades indígenas, la política lingüística debería incentivar la adquisición de la lengua de señas, del idioma oral-escrito de la comunidad indígena como segunda lengua y del español como tercera lengua. Reflexiona, a su vez, acerca de la educación bilingüe y bicultural en México. Propone que para que el bilingüismo esté bien desarrollado, deben participar como docentes sordos adultos con un muy buen manejo de la LSM y exhorta a la familia de los niños sordos a aprender la lengua de señas y a participar de las actividades propuestas por la comunidad sorda local. Así también, admite la dificultad que implica la enseñanza de la lecto escritura del español como segunda lengua a esta población y sostiene que deben realizarse nuevas investigaciones al respecto.

\section{Dificultades sintácticas}

Stockseth Danzak (2002) considera que los sordos tienen obstáculos a la hora de comprender textos y, más específicamente, en cuanto a la comprensión de estructuras sintácticas. Cita estudios en habla inglesa que indican que los sordos mostraron menos dificultades en estructuras cuyos constituyentes explícitos eran SVO, mientras que tuvieron mayor dificultad en estructuras con categorías vacías o con una estructura distinta a SVO. Por su parte, mostraron déficits en estructuras de disyunción y alternancia, oraciones subordinadas de relativo, frases de infinitivo y de gerundio y pronombres (Berent, 1988; Paul y Quigley, 1994, citados en Stockseth Danzak, 2002). En un estudio realizado por la autora, orientado a descubrir el nivel de comprensión sintáctica de niños 
y adultos sordos chilenos con algún grado de competencia lectora, descubrió el grupo de niños contaba con una comprensión global de $37 \%$ mientras que el grupo de adultos, con un $48 \%$, mostrando mayor facilidad para la comprensión de estructuras SVO (80\% en el grupo de niños y $100 \%$ en el grupo de adultos) y mayor dificultad para las estructuras ovs ( $18 \%$ alumnos, $20 \%$ adultos), interpretándola como de estructura Svo. Descubre también que ambos grupos comprenden mayoritariamente las flexiones de verbos de segunda y tercera persona del singular, aunque todos presentaron gran dificultad con la flexión verbal de la primera persona del singular. Si bien pudieron comprender los verbos en subjuntivo (mejor desempeño entre los adultos que entre los niños), presentaron serias dificultades en la comprensión del condicional. Mostraron, a su vez, problemas para comprender cláusulas relativas y pronombres acusativos anafóricos. La autora llega a la conclusión de que el procesamiento que hacen los sordos -sobre todo los niños- es puramente léxico sin hacer uso de información sintáctica: en una frase visualizan sustantivos, adjetivos y verbos, dejando de lado las palabras funcionales. En esto coinciden Domínguez Gutiérrez y Alegría (2009), quienes sostienen que las estrategias lectoras de los sordos suelen estar vinculadas con la identificación únicamente de palabras clave aquellas con contenido semántico propio, como sustantivos y verbos- y tienen claras dificultades con las palabras funcionales.

A su vez, Domínguez Gutiérrez y Soriano Bozolongo (2009) remarcan la importancia que tiene la inclusión de ejercicios que estimulen el aprendizaje de la sintaxis en la educación del sordo, especialmente en cuanto al orden de las palabras, la inclusión de palabras funcionales y los signos ortográficos, vinculándolos también con aspectos de índole semántico.

\section{La logogenia, una posible respuesta a las dificultades sintácticas de los sordos}

En el apartado anterior se hizo alusión a la falta de procesamiento sintáctico en los sordos y a la necesidad de generar espacios de ejercitación de las habilidades sintácticas.

Ante esta situación, surge el interrogante acerca de cómo trabajar estos aspectos en la comunidad sorda. Una posible alternativa es el método logogenia, que fue creado por Radelli (1985, 1986, 1993, 1994 a, 1994b, 1997, 1999, 2000) y que está basado en la gramática generativa (Chomsky, 1957). Tiene como objetivo estimular a sordos e hipoacúsicos en la comprensión del lenguaje escrito, en una primera etapa, y en la producción de lenguaje escrito, en una segunda etapa. Radelli (2000) considera que los sordos prelingüísticos escasamente llegan a tener competencia lingüística en la lengua del país que habitan -sí tienen competencia lingüística en LS siempre y cuando hayan sido expuestos a la misma desde una edad temprana y en contacto con hablantes competentes en LS-, aunque puedan tener competencia comunicativa. Partiendo de la base de que adquirir una lengua es un proceso diferente que aprenderla, el método de la logogenia tiene como objetivo activar en los sordos un proceso de adquisición de cualquier lengua histórico-vocal que sea similar al proceso natural de adquisición de la lengua que se desarrolla en cualquier niño desde sus primeros años de vida (Radelli, 2000). La logogenia parte de la noción chomskiana de que la oración no es una cadena de palabras, sino que las oraciones se asemejan a árboles, que se ramifican en sintagmas, nominales, preposicionales, verbales (Chomsky, 1957). A partir de estas nociones básicas, el método 
busca ofrecer a los sordos un input lingüístico significativo mediante oposiciones expresadas a través de pares mínimos de oraciones que se diferencian por un solo elemento. Estos se presentan en forma de órdenes escritas que el niño debe ejecutar. Cuando no sabe hacerlo, se enseña la orden a otra persona, que la ejecutará en presencia del niño. Se trabaja con las estructuras sintagmáticas del generativismo (Bosque y Gutiérrez-Rexach, 2009; Eguren y Fernández Soriano, 2004), pero, en la práctica, no se deben usar los términos como "sujeto", "predicado", etc., ya que lo que se pretende es sumergir al niño en el lenguaje escrito, para que pueda adquirirlo, no enseñarle con etiquetas metalingüísticas.

Las oposiciones que se le pueden presentar al alumno son léxicas ("Dame la manzana", "Dame la pera", "Agarrá la pera"); en el orden de los elementos ("Dame la mano", "La mano dame"); en la forma en que uno de los elementos transmite información sintáctica ("Dibujá una pera y una manzana y recortala", "Dibujá una pera y una manzana y recortalas"); ausencia y presencia de un elemento ("Señalá el auto verde y el rojo", "Señalá el auto verde y dame el rojo"); sustitución de un elemento por otro ("Agarrá la flor rosa y la violeta", "Agarrá la flor rosa o la violeta"). También pueden realizarse estas oposiciones para focalizar el nivel ortográfico y de puntuación.

En una primera etapa, el logogenista evalúa el nivel de comprensión sintáctica escrita mediante órdenes simples y órdenes complejas (por ejemplo, que incluyan preposiciones o cláusulas copulativas o comparativas). Si el alumno comprende órdenes simples se le pueden presentar tanto órdenes simples como complejas aleatoriamente. En caso de que no comprenda órdenes simples, el método comienza por entrenar la comprensión más básica, para luego pasar a la siguiente etapa. Cuando se mezclan oraciones simples y complejas, se trata de incluir preposiciones y nexos de distinto tipo, hasta que el alumno sea capaz de comprender órdenes que incluyen cláusulas subordinadas. Cuando logra resolver este tipo de órdenes, se puede pasar a una segunda etapa del método, que incluye el diálogo escrito entre el logogenista y el alumno, esperando que la producción escrita sea gramaticalmente correcta. En caso de que se presentaran algunos casos de agramaticalidad, se resuelven por escrito, en el mismo diálogo. Una vez que los diálogos fluyen de manera gramatical, se puede pasar a la comprensión de textos simples, cuyos contenidos serán evaluados mediante el diálogo por escrito entre el logogenista y el alumno, hasta llegar a la producción gramatical de textos. Cuando se llega a esta última etapa sin dificultades, la intervención ha terminado. Se estima que, en adultos, la intervención puede llevar alrededor de dos años (con tres sesiones semanales de logogenia), mientras que en niños es más prolongado, porque se encuentran en etapa de adquisición de procesos lingüísticos.

A partir de la creación de este método, muchos han sido los grupos que lo han implementado ${ }^{5}$, y lo siguen haciendo en México, Italia, Argentina, Colombia y España, pero son pocos los que han realizado investigaciones en torno del mismo. A pesar de esto, se puede mencionar que sí existen algunos estudios, en su mayoría de corte cualitativo, en las que los logogenistas-investigadores se abocan a la observación cualitativa de la propia práctica y a los estudios de caso. Entre estos estudios, podemos mencionar los de Pool Westgaard (2002, 2006), que se aboca a la definición de la naturaleza biológica del

\footnotetext{
${ }^{5}$ Cruz Aldrete (2009) menciona al método como una posibilidad, indicando que se lleva a cabo un programa de logogenia como parte de la educación bilingüe de niños sordos en el grupo educativo TESSERA en México.
} 
lenguaje y posteriormente analiza prácticas de logogenia tempranera -previa a la alfabetización-, con argumentos, más que con resultados, acerca de la importancia de exponer a los niños sordos al lenguaje escrito incluso antes de ser alfabetizados. Su principal argumento es que así como los niños normo-oyentes están expuestos al lenguaje desde que nacen y esto ayuda a la normal adquisición de la lengua, en el caso de los sordos, el hecho de estar expuestos al lenguaje escrito desde edad temprana, ayudará a su adquisición. Vale aclarar que, desde nuestro punto de vista, esta hipótesis es contraria al generativismo ya que, desde este marco teórico, la adquisición de una lengua natural no puede ser equiparada con la adquisición de una tecnología artificial -como lo es la escritura-. Otros estudios son el de Musola (2000), quien, a través del análisis de casos, indaga sobre la importancia y el rol que cumplen las categorías funcionales en el método, llegando a la conclusión de que estas son el pilar fundamental de la logogenia y que es mediante ellas que el sordo accede a la comprensión sintáctica; otras investigaciones desarrollan, en una primera etapa, la inmersión en el lenguaje que viven los niños sordos a través de la logogenia (Franchi, 1998; Franchi y Radelli, 2000), y luego, en una segunda etapa, se aborda el caso de un sordo profundo sin acceso a lengua de señas, y su adquisición, a través del método, del verbo "ser" como copulativo y como auxiliar (Franchi, 2004), llegando a la conclusión de que comprende y produce el verbo "ser" como auxiliar y como copulativo de manera correcta en un 37\% durante el proceso. Así también, Fernández Botero (2005) analiza casos de logogenización divididos en cuatro grupos: sordos oralizados con competencia oral moderada, sordos que al inicio del proceso de logogenización no tenían competencia comunicativa ni en lengua oral, ni en lengua escrita ni en lengua de señas, sordos en proceso de oralización pero sin competencia en lengua oral, y sordos que tienen competencia comunicativa solo en lengua de señas, notando mejorías en todos los grupos. Por su parte, a partir del análisis de un caso de logogenización de manera longitudinal -a lo largo de dos años de implementación del método-, Salas y Velazco (2015) elaboran un material orientado al seguimiento del proceso y ejercicios para adaptar a las sesiones de logogenia. A su vez, Salas (2015) establece vínculos entre la logogenia y las neurociencias del lenguaje, ya que, mientras estas últimas buscan conocer aspectos relacionados con el procesamiento del lenguaje en personas que presentan patologías lingüísticas mediante el estudio de casos y la logogenia funcionaría como una posible solución a los problemas de procesamiento lingüísticos intrínsecos a la sordera.

En el marco de investigaciones cualitativas, se destacan también estudios anteriores de nuestra autoría, en los que se compararon producciones espontáneas iniciales y producciones espontáneas luego del proceso de logogenización en cinco casos estudiados en profundidad, encontrando mejorías en la concordancia gramatical, en la compleción de cláusulas coordinadas y subordinadas, entre otras (Hael, 2015a). Asimismo, se han realizado estudios cualitativos de sesiones de logogenia abocadas a la comprensión de hiperónimos e hipónimos (Hael, 2015b) y a la concordancia gramatical sustantivo-artículo, sustantivo-adjetivo y sustantivo-verbo (Hael, 2016), registrándose mejoras a lo largo de las sesiones en ambos casos. Por último, se realizó un estudio que aborda los posibles avances en comprensión lectora por parte de sordos asistentes a universidades públicas de Argentina a partir del método (Alonso, Bordino y Hael, 2016).

Además de los estudios cualitativos acerca de la aplicación del método, existe una investigación (Vidal Matosas, 2014) que aborda cuantitativamente y comparativamente el nivel de desarrollo de la comprensión lectora en sordos adultos antes del inicio del proceso 
de logogenización y luego de la intervención a través del método durante siete meses. Esta investigación presenta como resultados la mejora de la comprensión sintáctica en su población de estudio, medida a través de los tests TSA (que se enfoca en la comprensión morfosintáctica) y ECLE (que se enfoca en la comprensión lectora): toda la muestra presentó mejoras con respecto a su punto de partida, si bien existieron puntos en los que se debía seguir trabajando mediante el proceso de logogenización.

Nos parece válido mencionar, asimismo, que actualmente en Italia se implementa un programa de logogenia para la enseñanza de italiano como segunda lengua a personas oyentes que no son hablantes nativos de la lengua italiana (Bonanno, Delliri, Dolza y Maglione, 2013). Esto aporta evidencia de que la logogenia no es contraria al bilingüismo y de hecho puede emplearse como un método alternativo o más bien accesorio ${ }^{6}$ para el aprendizaje de cualquier lengua alfabética como segunda lengua.

\section{Conclusiones}

En este trabajo hemos desarrollado los aportes teóricos más relevantes en español acerca de la lectura de la población sorda. Hemos podido comprobar que el bilingüismo es la corriente más difundida en la actualidad, que defiende la adquisición de la lengua de señas como primera lengua y del español como segunda lengua, en contraposición al oralismo que era la corriente prioritaria en décadas pasadas- que plantea la adquisición del español como única lengua. A su vez, dimos cuenta de la existencia de diferentes corrientes en el ámbito del bilingüismo, que abarca la posibilidad de apropiarse del español solo en su variante escrita o adquirirlo en sus versiones escrita y oral. Muchas de las investigaciones relevadas para este artículo están a favor de esta segunda posibilidad, ya que consideran que el desarrollo de la conciencia fonológica es fundamental a la hora de aprender a leer, y en el caso de sordos - en contextos bilingües- se combina con la lectura labial, con la dactilología, con la palabra complementada o, en casos más extremos, con el implante coclear. Por su parte, no hemos encontrado resultados de investigaciones que demuestren cómo se procese la lectura sin acceso a la conciencia fonológica, parte de la propuesta del bilingüismo tradicional. En este sentido y ante la evidencia, consideramos que el bilingüismo amplio, con acceso al conocimiento del español escrito y oral es apropiado para la adquisición de la lectura. En este aspecto cabe aclarar que creemos que la oralidad es solo un vehículo a la posibilidad de activar la conciencia fonológica que, según se ha comprobado, tiene incidencia positiva en los procesos lectores de la comunidad sorda, y no un intento por reemplazar la lengua de señas. El acceso a lo oral, desde nuestra perspectiva, debe estar orientado únicamente en función de la lectura.

En esta línea, pensamos que la educación del sordo debe ser verdaderamente bilingüe, de respeto por su lengua materna, la de señas, pero con iguales posibilidades de acceso al español -en caso de países de habla hispana-. Coincidimos con Sánchez Avedaño (2005) y con Lissi, Svartholm y González (2012) en la necesidad de que adultos sordos participen en el ámbito educativo de la comunidad, así como también en la importancia de su formación: quienes enseñen a leer y a escribir a sordos en lengua española tienen que ser

\footnotetext{
${ }^{6}$ Creemos que no se puede emplear logogenia como único método de enseñanza de segunda lengua -sobre todo para oyentes- porque el desarrollo de competencia lingüística y comunicativa oral en esos casos es también fundamental. Consideramos, sí, que la logogenia ofrece aportes significativos para el aprendizaje de las estructuras gramaticales y para la lectura y escritura de segundas lenguas.
} 
competentes en ambas lenguas. Así también, la lengua de señas debería ser estudiada de manera completa, como una asignatura independiente y atendiendo a todos los niveles del lenguaje: fonológico, morfológico, léxico, sintáctico, semántico y pragmático. Debe ser el vehículo de enseñanza de todas las asignaturas de la currícula escolar -abordadas por docentes competentes tanto en la disciplina particular como en la lengua de señas- y no retransmitidas por intérpretes que simplemente traducen lo que dice el docente especialista en la materia, como refieren Larrinaga y Peluso (2007). Así también, la lengua de señas debe ser el vehículo a través del cual se enseñe el español, tendiente a facilitar, por ejemplo, la comprensión de vocabulario, la explicación de conceptos o la comparación entre estructuras gramaticales de ambas lenguas, pero para ello, es fundamental que el nivel lingüístico en lengua materna sea elevado. En cuanto a las estructuras gramaticales del español, y tal como hemos relevado, la sintaxis es uno de los aspectos que mayores dificultades generan en la comprensión lectora de las personas sordas. Esas investigaciones, en términos generales, apuntan a que los sordos llevan a cabo un procesamiento puramente léxico del lenguaje, fijándose en palabras familiares y no en las palabras clave de los textos que leen. No suelen reparar en las palabras funcionales y las cláusulas más complejas les generan complicaciones en la lectura. Ante esta certeza, diversos autores indican que deben proponerse ejercicios específicos para entrenar la sintaxis de la lengua escrita en sordos. En esta línea de estudios se puede mencionar al método logogenia, que expone a los sordos a la sintaxis del español a través de órdenes que deben ejecutar y que se suceden con un solo cambio sintáctico a la vez, para proporcionar pares mínimos que les permitan advertir los contrastes. Algunas de las investigaciones relevadas en relación con este método aportan resultados positivos en cuanto a la adquisición de sintaxis. Como se ha hecho mención en el desarrollo del artículo, la logogenia se ha expandido hacia la metodología de enseñanza de italiano como segunda lengua para personas oyentes que no son hablantes nativos de ese idioma. En este sentido, consideramos que puede ser un método que complemente la enseñanza de la lectura en español como segunda lengua en el caso de sordos, atendiendo específicamente al aspecto sintáctico, que, tal como se ha analizado con anterioridad, es uno de los que mayores dificultades genera en esta población.

A partir de las consideraciones expuestas en este artículo y atendiendo a que los docentes manifiestan que la baja comprensión lectora incide negativamente en el desarrollo de las demás asignaturas escolares -incluso en escuelas de sordos-(Larrinaga y Pelusso, 2007), pensamos que no solo es importante, sino imprescindible el acceso a vocabulario, a la conciencia fonológica y a las estructuras sintácticas del español para la comprensión lectora en esta población, que tiene incidencia en la trayectoria educativa en su totalidad. El bilingüismo debería sistematizar el abordaje de la lectura y la escritura y formar docentes capaces de llevar a cabo esa tarea -y la tarea de enseñanza de todas las asignaturas- de mano de personas competentes en las disciplinas y en LS, así como también tiene el desafío de aplicar métodos que hayan demostrado su eficacia o bien generar nuevas metodologías que permitan el acceso a la lectura por parte de los sordos. La importancia que tiene la lectura para el acceso de los sordos a los estudios primarios, secundarios y superiores radica en que es uno de los principales vehículos del saber y, por ende, una de las mejores posibilidades de acceder y generar conocimiento sobre cualquier temática. Saber leer y comprender lo que se lee tiene un impacto fundamental en el desarrollo cognitivo y es un derecho humano básico: El ámbito educativo debe luchar por el acceso de los sordos no solo a estar alfabetizados en el sentido de decodificar lo escrito, sino 
fundamentalmente a poder comprender lo que leen y poder escribir de manera autónoma. En este sentido, y en concordancia con Domínguez Gutiérrez y Soriano Bozolongo (2009), que los sordos puedan acceder a la palabra escrita de manera cabal permite la democratización del conocimiento y el acceso verdaderamente inclusivo a la educación y a la participación ciudadana en su totalidad.

\section{Referencias}

Alegría, J. (2003). Deafness and reading. En T. Nunes y P. Bryant (Eds.). Handbook of children's literacy (pp. 459-489). Dordrescht: Kluwer Academic Publishers.

Alonso, A., Bordino, G. y Hael, M. V. (2016). Aportes de la logogenia a la inclusión de estudiantes con discapacidad auditiva en la educación superior. En M. Schorn (Coord.), Libro de resúmenes del congreso internacional salud mental y sordera "Inclusión: El desafio que plantea el cambio de paradigma” (pp. 728-739). Buenos Aires: Facultad de Psicología de la Universidad de Buenos Aires.

Augusto, J. M., Adrián, J. A., Alegría, J. y Martínez de Antoñana, R. (2002). Dificultades lectoras en niños con sordera. Psicothema, 14(4), 746-753.

Bonanno, S., Delliri, F., Dolza, E., Maglione, E. (2013). Il manuale di lingua italiana per sordi stranieri. Roma: Cartman.

Bosque, I. y Gutiérrez-Rexach, J. (2009). Fundamentos de sintaxis formal. Madrid: Akal.

Chomsky, A. N. (1957). Estructuras sintácticas. Ciudad de México: Ed. Siglo XXI.

Cruz Aldrete, M. (2009). Reflexiones sobre la educación bilingüe intercultural para el sordo en México. Revista Latinoamericana de Educación Inclusiva, 3(1), 133-145.

Cummins, J. (1984). Bilingualism and special education: Issues in assessment and pedagogy. Clevedon: Multilingual Matters.

Domínguez Gutiérrez, A. B. y Alegría, J. (2009). Los alumnos sordos y la lengua escrita. Revista Latinoamericana de Educación Inclusiva, 1(3), 85-111.

Domínguez Gutiérrez, A. B. (2003). ¿Cómo acceden los alumnos sordos al lenguaje escrito? Enseñanza, 21, 201-218.

Domínguez Gutiérrez, A. B., Pérez Sánchez, I. y Soriano Bozalongo, J. (2007). El aprendizaje de la lectura de los alumnos sordos: resultados preliminares. Enseñanza, 25, 93-110.

Domínguez Gutiérrez, A. B. y Soriano Bozalongo, J. (2009). Mecanismos de lectura empleados por personas sordas adultas consideradas como buenas lectoras. Bordón, 61(4), 9-20.

Eguren, L. y Fernández Soriano, O. (2004). Introducción a una sintaxis minimista. Madrid: Gredos.

Ellis, A. y Young, A. (1992). Neuropsicología cognitiva humana. Barcelona: Masson, S. A.

Fernández Botero, E. L. (2005). Reflexiones acerca de la logogenia a partir de una experiencia directa con ella. Recuperado de http://www.dimecolombia.org/articulo5.html

Figueroa, V. y Lissi, M. R. (2005). La lectura en personas sordas: Consideraciones sobre el rol del procesamiento fonológico y la utilización del lenguaje de señas. Estudios Pedagógicos, 31(2), 105-1 19. https://doi.org/10.4067/S0718-07052005000200007

Franchi, E. (1998). Nel mondo dei sordi. Un camino verso il linguaggio. Il ruolo delle cattegorie funzionali e la logogenia. Venecia: Università Ca' Foscari Venezia. 
Franchi, E. (2004). Piena competenza e assenza di competenza lingüística: Una distinzione messa in evidenza della logogenia. Essere copula e ausiliare in Italiano infantile e in un sordo profondo prelinguale non segnante. Florencia: Universitá degli Studi di Firenze.

Franchi, E. y Radelli, B. (2000). La logogenia y el desarrollo lingüístico de los sordos. Diario de Campo. Boletín Interno de los Investigadores del Área de Antropología, 28, 367-382.

Grosjean, F. (2000). El derecho del niño sordo a crecer bilingüe. El Bilingüismo de los Sordos, 1(4), 15-18.

Hael, M. V. (2015a). Producción escrita espontánea de pacientes hipoacúsicos. En R. I. Castaldo (Ed.), Actas del IV Congreso de Psicología del Tucumán. "Cultura y Subjetividad: La Psicología Interpelada", (pp. 343-358). Facultad de Psicología de la Universidad Nacional de Tucumán, San Miguel de Tucumán.

Hael, M. V. (2015b). Comprensión de hiperónimos por parte de sordos a través del método logogenia. Revista Argentina de Ciencias del Comportamiento, 1, 104-105.

Hael, M. V. (2016). Comprensión de elementos de concordancia sintáctica por parte de sordos a través del método logogenia. En Y. Hipperdinger (Ed.) Libro de resúmenes del XV congreso de la sociedad argentina de lingüística: $A 100$ años de la publicación del curso de lingüística general (pp. 106-107). Bahía Blanca: Sociedad Argentina de Lingüística y Universidad Nacional del Sur.

Henao Álvarez, O., Ramírez Salazar, D. y Medina Medina, M. (2004). Potenciando la capacidad lectora de los sordos con el apoyo de nuevas tecnologías. Lectura y Vida, 25(4), 18-25.

Herrera Fernández, V. (2009). En busca de un modelo educativo y de lectura coherente con las necesidades educativas especiales de los estudiantes sordos. REXE. Revista de Estudios y Experiencias en Educación, 8(16), 11-24.

Larrinaga, J. A. y Peluso, L. (2007). Educación bilingüe de los sordos: Consideraciones acerca de la escritura, procesamiento del conocimiento y rol docente. En UBA. (Ed.), La investigación en psicología, su relación con la práctica profesional y la enseñanza: Memorias de las XIV Jornadas de investigación, tercer encuentro de investigadores de psicología del Mercosur (pp. 463-466). Buenos Aires: Ediciones de la Facultad de Psicología de la Universidad de Buenos Aires.

Lissi, M. R., Svartholm, R. y González, M. (2012). El enfoque bilingüe en la educación de sordos: Sus implicancias para la enseñanza y el aprendizaje de la lengua escrita. Estudios Pedagógicos, 38(2), 299-320. https://doi.org/10.4067/S07 18-07052012000200019

Martínez de Antoñana, R. M. y Augusto Landa, J. M. (2002). La lectura en los niños sordos: El papel de la codificación fonológica. Anales de Psicología, 18(1), 183-195.

Musola, D. (2000). La logogenia. Viaggio al centro della lingua: La nascita della lingua nei sordi. Padua: Universitá degli Studi di Padova.

Peluso, L. y Vallarino, S. (2014). Panorámica general de la educación pública de los sordos en Uruguay a nivel de Primaria. Psicología, Conocimiento y Sociedad, 4(2), 211-236.

Pool Westgaard, M. (2002, octubre). El lenguaje humano y su naturaleza biológica. Conferencia presentada en el acto de Inauguración del Diplomado en Logogenia, Servicios de Educación Integrada del Estado de México. Escuela Nacional de Antropología e Historia, Ciudad de México.

Pool Westgaard, M. (2006). La logogenia tempranera: ¿Cómo y por qué? En F. Barriga Puentes (Comp.), Actas del II Congreso Internacional de Logogenia (pp. 204-221). Ciudad de México: Museo Nacional de Antropología. 
Radelli, B. (1985). La ambigüedad: Un rasgo significativo para el análisis sintáctico. Ciudad de México: Instituto Nacional de Antropología e Historia.

Radelli, B. (1986). El cuál y el cómo en la sintaxis del español. En B. Garza Cuarón y P. Levy (Eds.), Homenaje a Jorge A. Suárez (pp. 437-445). Ciudad de México: El Colegio de México.

Radelli, B. (1993). Buscando configuraciones sintácticas y sus significados: pistas para neurólogos. En M. C. Muntzel y R. Bruna (Coords.), Homenaje a Leonardo Manrique (pp. 125-134). Ciudad de México: INHA.

Radelli, B. (1994a). Naturaleza del lenguaje y problemas para la rehabilitación de los niños sordos. En Z. Estrada Fernández, M. Figueroa Esteba y G. López Cruz (Eds.), Memorias del segundo encuentro de lingüistica en el noroeste (pp. 189-213). Sonora: Universidad de Sonora.

Radelli, B. (1994b). Agramaticalidad, ambigüedad sintáctica y metáfora: Criterios e instrumentos para evaluar la adquisición de la competencia lingüística. Revista Dimensión Antropológica, $1(1), 79-102$.

Radelli, B. (1997). Significados sintácticos. En M. Pool Westgaard (Ed.), Estudios de lingüística formal (pp. 237-256). Ciudad de México: El Colegio de México.

Radelli, B. (1999). La logogenia en el desarrollo de los sordos. En A. Pérez Sanz (Ed.), Actas del XV congreso nacional FEPAL: Elección de métodos y sistemas en la educación del sordo y patologías del lenguaje del siglo XXI (pp. 169-190). A Coruña: Universidad de A Coruña.

Radelli, B. (2000). Una nueva aplicación de la lingüística: La logogenia. En A. Álvarez González y colabordores (Comps.), Memorias del sexto encuentro internacional de lingüística del noroeste (pp. 51-72). Ciudad de México: Universidad de Sonora.

Salas, P. (2015). Sordera y lenguaje: Neurociencias y logogenia. Córdoba: Editorial Brujas.

Salas, P. y Velazco, M. (2015). Logogenia para niños sordos. Material para el seguimiento y desarrollo lingüístico del español rioplatense. Córdoba: Editorial Brujas.

Sánchez Avedaño, C. (2005). El español y el LESCO en el marco de la enseñanza de una segunda lengua para las personas sordas en Costa Rica. Revista Educación, 29(2), 217-232. https://doi.org/10.15517/revedu.v29i2.2249

Skliar, C. (1998). Bilinguismo y biculturalismo: Un análisis sobre las narrativas tradicionales en la educación de los sordos. Revista Brasileira de Educação, 8, 44-57.

Stockseth Danzak, R. (2002). Comprensión de la sintaxis española por lectores sordos chilenos. Revista Signos, 35(51), 271-290.

Vidal Matosas, G. P. (2014). Logogenia. Análisis de su implementación en adultos con déficit auditivo perlocutivo. Trabajo de Fin de Máster. Universidad de Extremadura, Badajoz.

Zambrano, L. (2002). El español escrito como segunda lengua para sordos ¿Utopía o realidad? Lingua Americana, 4(11), 65-76.

\section{Breve CV de la autora}

\section{María Virginia Hael}

Licenciada en Letras y Doctora en Humanidades (área Letras) por la Facultad de Filosofía y Letras de la Universidad Nacional de Tucumán. Diplomada en Logogenia por el Colegio de Logogenistas de México. Becaria posdoctoral de temas estratégicos del Consejo Nacional de Investigaciones Científicas y Tecnológicas (CONICET) con el tema "Comprensión y producción sintáctica de sordos a través del método logogenia". Ex 
becaria doctoral de CONICET. Ex becaria MAEC-AECID en Cooperación lingüística internacional con sede en la Academia Argentina de Letras. Docente de Análisis y Producción del Discurso Especializado Escrito en Español del Traductorado en Inglés en el IES en Lenguas Vivas Juan Ramón Fernández. ORCID ID: https://orcid.org/oooo0002-7573-7846. Email: virginiahal@gmail.com 